\title{
BMJ Open Impact of hierarchical hospital reform on patients with diabetes in China: a retrospective observational analysis
}

\author{
Junfang Xu, ${ }^{1}$ Xin Wang (D) , ${ }^{2}$ Hongying Hao, ${ }^{3}$ Jian Wang (D) , 4,5 \\ Stephen Nicholas ${ }^{6,7,8,9}$
}

To cite: $\mathrm{Xu}$ J, Wang $\mathrm{X}$, Hao $\mathrm{H}$, et al. Impact of hierarchical hospital reform on patients with diabetes in China: a retrospective observational analysis. BMJ Open 2021;11:e041731. doi:10.1136/ bmjopen-2020-041731

- Prepublication history for this paper is available online. To view these files, please visit the journal online (http://dx.doi org/10.1136/bmjopen-2020041731).

Received 24 June 2020 Revised 02 March 2021 Accepted 03 March 2021
Check for updates

(C) Author(s) (or their employer(s)) 2021. Re-use permitted under CC BY-NC. No commercial re-use. See rights and permissions. Published by BMJ.

For numbered affiliations see end of article.

Correspondence to

Dr Jian Wang;

00032954@whu.edu.cn

\section{ABSTRACT}

Objectives We assess whether China's diabetes pilot hierarchical diagnosis and treatment reforms shifted patient healthcare-seeking behaviour towards primary health institutions (PHIs) and away from secondary and tertiary hospitals. From the patients' perspective, we evaluate whether the hierarchical system saw the decline of average hospital cost, out-of-pocket (0OP) expenses and the increase of reimbursement rate in PHIs. From the health system's perspective, we also assessed whether the share of PHIs in total costs, total visits and reimbursement rate increased and the share of secondary and tertiary hospitals decreased.

Methods Data were collected from the health insurance bureau of 11 cities in Shandong Province, China between 2015 and 2017, which included 9118518 outpatient visits and 622739 inpatient visits. For both inpatients and outpatients and the health system, we analysed health services-seeking characteristics including hospital costs hospital visits, 00P expense and reimbursements of hospital costs. Binary logistic regression was conducted to analyse the influencing factors of seeking $\mathrm{PHI}$ health services.

Results PHIs remained the lowest hospital cost provider, but average hospital costs declined across all three healthcare levels of PHIs, secondary hospitals and tertiary hospitals from 2015 to 2017. The hierarchical system aimed to shift patients to PHls, increasing PHIs' share of total hospital costs. However, the PHI share of total outpatient costs declined $12.0 \%$, while rising $15.0 \%$ in secondary hospitals, the opposite of the goal of the hierarchical medical system. Average outpatient visits rose roughly at the same rate in PHls $(5.1 \%)$ as secondary hospitals $(6.8 \%)$, with no evidence of a shift in patient visits between hospital levels over 2015-2017. Average inpatient visits fell across all levels of hospitals, with no significant difference in the rate of decline between PHIs (9.4\%) and secondary (7.5\%) and tertiary (7.8\%) hospitals. For outpatient and inpatient services, the binary logistic regression showed that over the 2015-2017 period patients with diabetes increasingly used higher level hospitals rather than PHIs $(p<0.05)$. The only success of the hierarchical medical system was the relative fall of 00P outpatient expenses, which fell more rapidly in PHIs (13.7\%) than secondary (5.0\%) and tertiary (3.5\%) hospitals. However, inpatient 0OP expenses fell only $2.2 \%$ for PHIs, less than half that of secondary $(5.5 \%)$ and tertiary (7.4\%) hospitals, the opposite of the aim of the hierarchical system reform.

\section{Strengths and limitations of this study}

This is one of the first studies to examine the effect of hierarchical medical system on diabetes treatment and management with a big database.

- The effects of China's diabetes pilot hierarchical diagnosis and treatment reforms were explored from both the patient and health system perspectives.

- The health insurance policies at the city level, including reimbursement regulations, provision of health services and status of the hierarchical medical system, were similar to the national-level policies, making our data broadly representative of China-wide experiences.

- The behaviour of patients with diabetes may diverge from the choices of patients with other illnesses, which might limit the generalisability of diabetes patient behaviour across the Chinese health system.

- Due to the limited variables in the medical insurance bureau, some information was not available for analyses, such as the severity of diabetes and comorbidities, which may influence the choice of hospital.

Conclusions The implementation of the hierarchical medical system for patients with diabetes did not achieve its goal of increasing PHI utilisation and decreasing secondary and tertiary hospital utilisation. Enhancing the utilisation of PHIs for diabetes and other patients requires further health reform, including educating patients on $\mathrm{PHI}$ use, further reforming the health insurance schemes, improving PHI facilities and encouraging referrals to PHIs from higher level hospitals.

\section{BACKGROUND}

As a major worldwide health problem, the number of people living with diabetes and its prevalence is growing globally, especially in low-income and middle-income countries. The International Diabetes Federation reported that 463 million adults, or 1 in 11 adults (20-79 years old), suffered from diabetes in $2019,{ }^{1}$ with $10 \%$ of the global health expenditure, or US $\$ 760$ billion, spent on diabetes and diabetes-related diseases. ${ }^{12}$ 
In 2019, China had the world's largest number of adults with diabetes, roughly 116.4 million, a large number of undiagnosed diabetes sufferers, perhaps as high as 65 million adults, and $15.5 \%$ of the adult Chinese population with pre-diabetes. ${ }^{2-4}$ In 2017 , diabetes and its complications accounted for $1.47 \%$ of China's total deaths, $4.56 \%$ total years lived with disability and $2.69 \%$ disabilityadjusted life years. ${ }^{2}$ China's diabetes health expenditures rose from US $\$ 0.25$ billion (or $0.07 \%$ of gross domestic product (GDP)) in 1993 to US $\$ 8.65$ billion (or $0.21 \%$ of GDP) in 2008, to US $\$ 109$ billion (or $0.79 \%$ of GDP) in 2019. ${ }^{15}$ These data point to the diagnosis, prevention and management of diabetes as an urgent challenge to China's health system.

China's current healthcare system is divided into three levels: primary health institutions (PHIs), secondary hospitals and tertiary hospitals. ${ }^{6} 7$ PHIs are tasked with providing preventive care, basic healthcare and rehabilitation services, and include township hospitals and community health service centres for daily management (including medication, follow-up care and health education) of chronic diseases, including diabetes. Usually affiliated with a medium-sized city, county or district, secondary hospitals, with 100-500 beds, provide comprehensive health services for patients including those with diabetes (covering examination, drug treatment and training for doctors), and include most county-level hospitals. Tertiary hospitals, with over 500 beds, are responsible for providing complex and specialist health services (such as treatment of patients with complicated and refractory diabetes with many complications) to multiple regions. Each level of China's healthcare facilities operates independently and competes for patients. Given the low patient confidence in PHI health technology and care, many patients attend secondary and tertiary level hospitals even for simple health problems and routine chronic disease diagnosis and treatment. ${ }^{3}$ This wasteful healthcare-seeking behaviour led to the overuse of higher level medical resources and patient complaints that it was both expensive and difficult to see a doctor, ${ }^{89}$ which caused low patient satisfaction with the health system. ${ }^{10-12}$

Against this background, China began to implement a hierarchical medical treatment system in 2015, with the aim to maximise the more efficient use of health resources, lessen the financial burden on patients and shift patient care choice to the appropriate level in the health system. In an ideal hierarchical medical treatment system, patients seek healthcare at an appropriate level hospital according to the urgency of their diseases. To make full use of PHIs and enable basic medical care at the community level, PHIs have added 'inpatient departments', especially for rehabilitation inpatients, to alleviate the shortages of beds at higher level hospitals and provide care closer to patients' homes. This was also one of the elements of the 'two-way referral' system to provide hierarchical diagnosis and treatment in China's medical reforms. However, evidence has shown that weak competence of healthcare provision at lower level hospitals and patients' preference for high-level hospitals are the main barriers to a hierarchical medical treatment system. ${ }^{13} 14$ Currently, there were two main strategies to shift patient healthcare choice, especially attracting patients to PHI health services, to lessen the financial burden on the patient and the health system. The first strategy was to improve the quality of healthcare provision in PHIs. ${ }^{15}$ The government increased the financial investment in PHIs significantly from $¥ 105.9$ billion (US $\$ 14.8$ billion) in 2013 to $¥ 180.8$ billion (US $\$ 25.3$ billion) in 2017 , with an average annual growth of $14.3 \%$, compared with the growth rate of $9.1 \%$ in secondary-tertiary hospitals. The increased investment in PHIs mainly focused on technical training, purchasing facilities and equipment, and subsidising secondary hospital doctors for seeing patients in PHIs. ${ }^{16}$ The second strategy was to change the compensation gap for hospital costs across different hospital levels, adjusting the insurance reimbursement rate to refund a higher proportion of out-of-pocket (OOP) hospital costs at PHIs than secondary and tertiary hospitals. ${ }^{17} 18$ Incentivising patients with common health issues to seek health services in PHIs, where the costs of treatment are on average $48 \%$ of outpatient costs at a tertiary hospital, optimises the allocation of hospital resources for China's health system. ${ }^{19}$

In fact, many countries have conducted related health reform to shift hospital care to primary care as an attempt to control the rising healthcare costs and reduce unnecessary referrals to hospitalisation services. ${ }^{1720} 21$ Limiting the growth of costly hospitalisation care was mainly achieved by increasing the care at PHIs. ${ }^{22}{ }^{23}$ The evidence has been mixed, with some evidence showing that these interventions reduced the utilisation of hospitalisation care $^{24}$; however, other studies show that PHI care for the less complex treatments did not show lower healthcare costs. 2526

In 2015, diabetes treatment and management were selected as the pilot for the implementation of the hierarchical medical system reforms in China. ${ }^{18}$ According to the analysis of the WHO, $65 \%-80 \%$ of patients with type 2 diabetes are in stable condition, only requiring maintenance treatment and self-management that can be provided by lower level PHI hospitals, while $15 \%$ of patients with diabetes are at medium risk and $5 \%$ at high risk, requiring more complex diabetes treatment in secondary and tertiary hospitals. ${ }^{19}$ The strategy of changing the insurance reimbursement rate aimed to shift patient hospital use from high-cost secondary-tertiary hospitals to low-cost PHIs. However, no large sample study has been conducted to examine the related effects in China. Against this background, to assess the implementation of China's hierarchical medical system, we analysed data on hospital medical costs, OOP expenses, reimbursement share (the percentage of reimbursements at each level of hospital in total reimbursements for all hospitals), and the number and share of visits by hospital level. Hospitals aimed to achieve the lowest hospital costs and patients aimed for the lowest OOP expenses. For 
patients with diabetes, a successful hierarchical medical system will increase the number of hospital visits, decrease OOP expenses and increase the reimbursement share in lower level PHI hospitals compared with higher level tertiary hospitals. The best outcome for the health system would be to lower average hospital costs, raise the rate of reimbursements and increase the share of PHI visits and hospital costs in total hospital costs, and decrease the share of secondary-tertiary hospital visits and costs.

\section{METHODS}

\section{Data sources}

Between 2015 and 2017, we collected data on patients with diabetes from the health insurance bureaus of 11 cities (Binzhou, Dongying, Heze, Jining, Laiwu, Taian, Weihai, Weifang, Yantai, Zaozhuang and Zibo) in Shandong Province in Eastern China. All data were from the databases of the health insurance bureaus; no patients were recruited and no personal data were collected. Permission was secured from the health insurance authorities, which required all the data should only be used for research. Before receiving access to the data set, researchers submitted the required data list to the health insurance bureaus, which determined that the variables did not breach patient confidentiality. After deleting records with missing data, the sample comprised 9118518 outpatients with diabetes and 622739 inpatients with diabetes. The data included information on sociodemographic characteristics (sex, age and residential city), clinical diabetes diagnosis based on the International Classification of Diseases-10, types of health insurance, number of visits to hospitals, hospital level (PHIs and secondary and tertiary hospitals), hospital costs, OOP expenses, insurance reimbursement rate (percentage of reimbursements in each hospital level's costs) and insurance reimbursement share (the percentage of reimbursements in total reimbursements for all hospitals) of patients with diabetes. Based on the per capita income, the level of residential city was divided into low, middle and high.

In 2015, Shandong Province merged the Urban Resident Basic Medical Insurance with the New Rural Cooperative Medical Insurance to the Integration of Urban and Rural Medical Insurance (IURMI) to improve reimbursement rates and reduce OOP expenses for rural residents and urban retired, unemployed, students and children. The urban employed were insured by the Urban Employee Basic Medical Insurance (UEBMI). Given China's two basic medical insurance schemes covered more than $95 \%$ of the population, ${ }^{27}$ patients recorded in the health insurance database included almost all outpatients and inpatients with diabetes during the study period. The health insurance policies at the city level, including reimbursement regulations, provision of health services and status of the hierarchical medical system, were similar to the national-level policies ${ }^{28}$ making our data broadly representative of China-wide experiences.

\section{Data analysis}

From both the patient and health system perspectives, we analysed the health service behaviour of patients with diabetes, measured by hospital medical costs, OOP expenses, reimbursement share and rate, and share of visits by level of hospital. From the patients' perspective, optimal health service-seeking behaviour was the highest average number of visits, lowest OOP expenses by inpatients and outpatients, and highest reimbursement rate at each level of hospital each year. From the health system's perspective, the aim was to encourage health service use at the lowest cost PHI hospital, with the highest reimbursement rate and highest share of visits. ${ }^{29}$

Frequencies and percentages were used to describe the sociodemographic information and number of hospital visits, and means were employed to describe the medical costs (including reimbursements and OOP expenses). All costs were converted to US dollar values in May 2020, when 1 Chinese yuan was equivalent to US\$0.1402. Binary logistic regression was conducted to analyse the influencing factors of whether patients with diabetes sought health services in PHI or non-PHI (secondary and tertiary) hospitals. The independent variables included sex, age, health insurance type, residential city income level and year of seeking health services. SPSS V.22.0 software was used to analyse all the data, with statistical significance set at $\mathrm{p}<0.05$.

\section{Patient and public involvement}

All data were from the databases of the health insurance bureaus; no patients were interviewed. Based on a list of variables submitted by the researchers, the medical insurance authorities provided anonymous data that did not breach patient confidentiality and did not identify individual patients.

\section{RESULTS}

Sociodemographic characteristics of patients with diabetes

Table 1 shows the growth of outpatients with diabetes between 2015 and 2017 from 2555472 to 3414741 and inpatients with diabetes from 189972 to 218343 . The characteristics of outpatients and inpatients with diabetes are shown in table 1 . Men accounted for $50.48 \%$ of outpatients and $46.18 \%$ of inpatients; $40.05 \%$ of inpatients and $32.27 \%$ of outpatients were under 60 years old; and IURMI patients accounted for $36.16 \%$ of outpatients and $57.75 \%$ of inpatients.

\section{Hospital costs, 00P expenses and reimbursement rate and share}

Figure 1 shows that the average outpatient and inpatients hospital costs and OOP expenses were lower in PHIs than in secondary-tertiary hospitals. The average outpatient hospital costs declined slightly across all hospital levels between 2015 and 2017, falling from $¥ 379.90$ (US\$53.26) to $¥ 331.23$ (US\$46.44) in PHIs; from ¥453.31 (US\$63.55) to $¥ 399.56$ (US\$56.02) in secondary hospitals; and 


\begin{tabular}{|c|c|c|c|c|}
\hline \multirow[b]{2}{*}{ Items } & \multicolumn{2}{|c|}{ Outpatients } & \multicolumn{2}{|c|}{ Inpatients } \\
\hline & $\mathbf{n}$ & $\%$ & $\mathbf{n}$ & $\%$ \\
\hline \multicolumn{5}{|l|}{ Gender } \\
\hline Male & 4603446 & 50.48 & 287596 & 46.18 \\
\hline Female & 4361484 & 47.83 & 327741 & 52.63 \\
\hline Missing & 153588 & 1.68 & 7402 & 1.19 \\
\hline \multicolumn{5}{|l|}{ Age } \\
\hline$\leq 30$ & 42595 & 0.48 & 10685 & 1.75 \\
\hline $31-40$ & 116006 & 1.29 & 26395 & 4.32 \\
\hline $41-50$ & 635607 & 7.09 & 60637 & 9.93 \\
\hline $51-60$ & 2100101 & 23.42 & 146953 & 24.06 \\
\hline $61-70$ & 3397362 & 37.89 & 202185 & 33.10 \\
\hline$\geq 71$ & 2675691 & 29.84 & 163991 & 26.85 \\
\hline \multicolumn{5}{|c|}{ Health insurance } \\
\hline IURMI & 3297188 & 36.16 & 359611 & 57.75 \\
\hline UEBMI & 5821330 & 63.84 & 263128 & 42.25 \\
\hline \multicolumn{5}{|l|}{ City level } \\
\hline High & 3964415 & 43.48 & 233693 & 38.03 \\
\hline Middle & 2741083 & 30.06 & 186154 & 30.29 \\
\hline Low & 2413020 & 26.46 & 194659 & 31.68 \\
\hline \multicolumn{5}{|l|}{ Year } \\
\hline 2015 & 2555472 & 28.03 & 189972 & 30.51 \\
\hline 2016 & 3148305 & 34.53 & 214424 & 34.43 \\
\hline 2017 & 3414741 & 37.45 & 218343 & 35.06 \\
\hline
\end{tabular}

IURMI, Integration of Urban and Rural Medical Insurance; UEBMI, Urban Employee Basic Medical Insurance.

from $¥ 515.58$ (US\$72.28) to $¥ 448.77$ (US\$62.92) in tertiary hospitals. While the decline in OOP expenses was greater in PHIs (from US\$21.67 to US\$18.70) than secondary (from US\$25.50 to US\$24.23) and tertiary (from US\$25.09 to US\$24.21) hospitals, OOP expenses fell across all hospital levels. The reimbursement rate, or the percentage of reimbursement in hospital costs at each level, also showed that the outpatient reimbursement rate fell slightly across different hospital levels, but remained highest in tertiary hospitals (from $62.83 \%$ to $58.32 \%$ ), with PHIs and secondary hospitals displaying the same reimbursement rate trend.

Inpatient hospital costs decreased slightly from $¥ 3863.40$ (US\$541.65) to $¥ 3368.08$ (US\$472.20) in PHIs, from $¥ 7149.84$ (US\$1002.41) to $¥ 6768.34$ (US\$948.92) in secondary hospitals, and from ¥10425.08 (US\$1461.60) to $¥ 8955.26$ (US\$1255.53) in tertiary hospitals. The inpatient reimbursement rate was highest in PHIs, but fell as a percentage of hospital costs (from $72.32 \%$ to $69.40 \%$ ), and remained steady in secondary (from $62.62 \%$ to $63.52 \%$ ) and tertiary (from $58.56 \%$ to $58.50 \%)$ hospitals. The OOP expenses faced by inpatients with diabetes fell slightly between 2015 and 2017, with OOP expenses lowest in PHIs (from US\$141.41 to US $\$ 138.33$ ), followed by secondary (from US $\$ 340.96$ to US $\$ 322.10$ ) and tertiary (from US $\$ 526.15$ to US $\$ 487.31$ ) hospitals.

Figure 2 shows that the share of secondary hospital outpatient costs was highest across all hospital levels, and rose from $43.15 \%$ in 2015 to $49.62 \%$ in 2017. PHIs' share (from $29.81 \%$ to $26.23 \%$ ) and tertiary hospitals' share (from $27.04 \%$ to $24.16 \%$ ) of total hospital costs fell slightly over 2015-2017. The share of OOP expenses in total hospital costs and the reimbursement share showed a similar trend to hospital costs, falling for PHIs and tertiary hospitals and rising in secondary hospitals. For inpatients, the share of inpatient hospital costs increased sharply in secondary hospitals from $47.05 \%$ to $53.76 \%$ between 2015 and 2017 and in tertiary hospitals from $40.87 \%$ to $45.59 \%$, but fell significantly in PHIs from $12.08 \%$ to $0.65 \%$. The share of inpatient OOP expenses and reimbursement share showed a similar trend, rising in secondary and tertiary level hospitals and falling in PHIs from 2015 to 2017.

\section{Hospital visits}

Figure 3 shows the average number of visits by outpatients and inpatients with diabetes. Across all hospital levels, outpatient visits increased, with the highest average number of visits in tertiary hospitals, but rising only slightly from 15.29 visits in 2015 to 15.62 visits in 2017, followed by PHI hospitals (rising from 14.28 visits in 2015 to 15.01 visits in 2017) and secondary hospitals (rising from 11.73 visits in 2015 to 12.53 visits in 2017). For inpatients, the average visits fell across all levels of hospitals from 2015 and 2017, with the greatest fall in tertiary hospitals (from 2.19 visits in 2015 to 2.02 in 2017), followed by secondary hospitals (from 1.46 visits in 2015 to 1.36 visits in 2017) and PHIs (from 1.28 times in 2015 to 1.17 times in 2017).

Figure 4 shows that the share of outpatient visits out of total outpatient visits to all hospitals was highest in secondary hospitals, rising sharply from $40.24 \%$ to $46.96 \%$ of all visits and fell from $37.77 \%$ in 2015 to $32.74 \%$ in 2017 in PHIs. For outpatients in tertiary hospitals and for inpatients at all hospitals, the share of diabetes visits remained largely unchanged.

\section{Influencing factors of patients with diabetes seeking health service in PHls}

Table 2 shows the influencing factors of whether patients with diabetes sought health service in PHIs versus secondary-tertiary hospitals. Female and older patients were more likely to seek health services in PHIs for both outpatient and inpatient services $(\mathrm{p}<0.05)$. For outpatient services, patients with UEBMI tended to use PHI health services, but chose higher level hospitals for inpatient services $(\mathrm{p}<0.05)$. During the last 3 years, patients with diabetes increasingly used higher level hospitals rather than PHIs regardless of outpatient and inpatient services $(\mathrm{p}<0.05)$. 

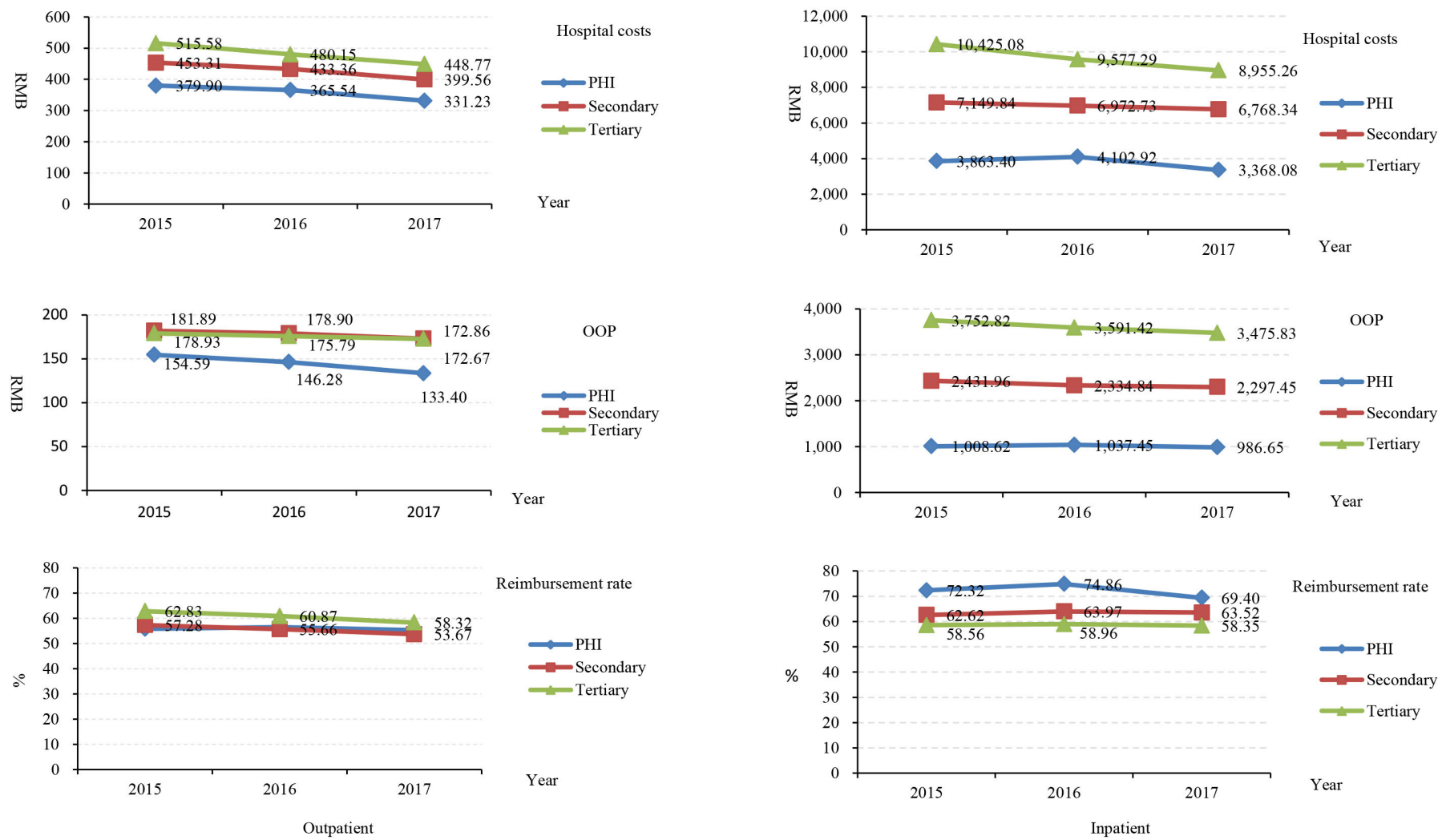

Figure 1 Average hospital costs, OOP expenses and reimbursement rate by level of hospital. OOP, out-of-pocket; PHI, primary health institution; $\mathrm{RMB}$, renminbi (¥).
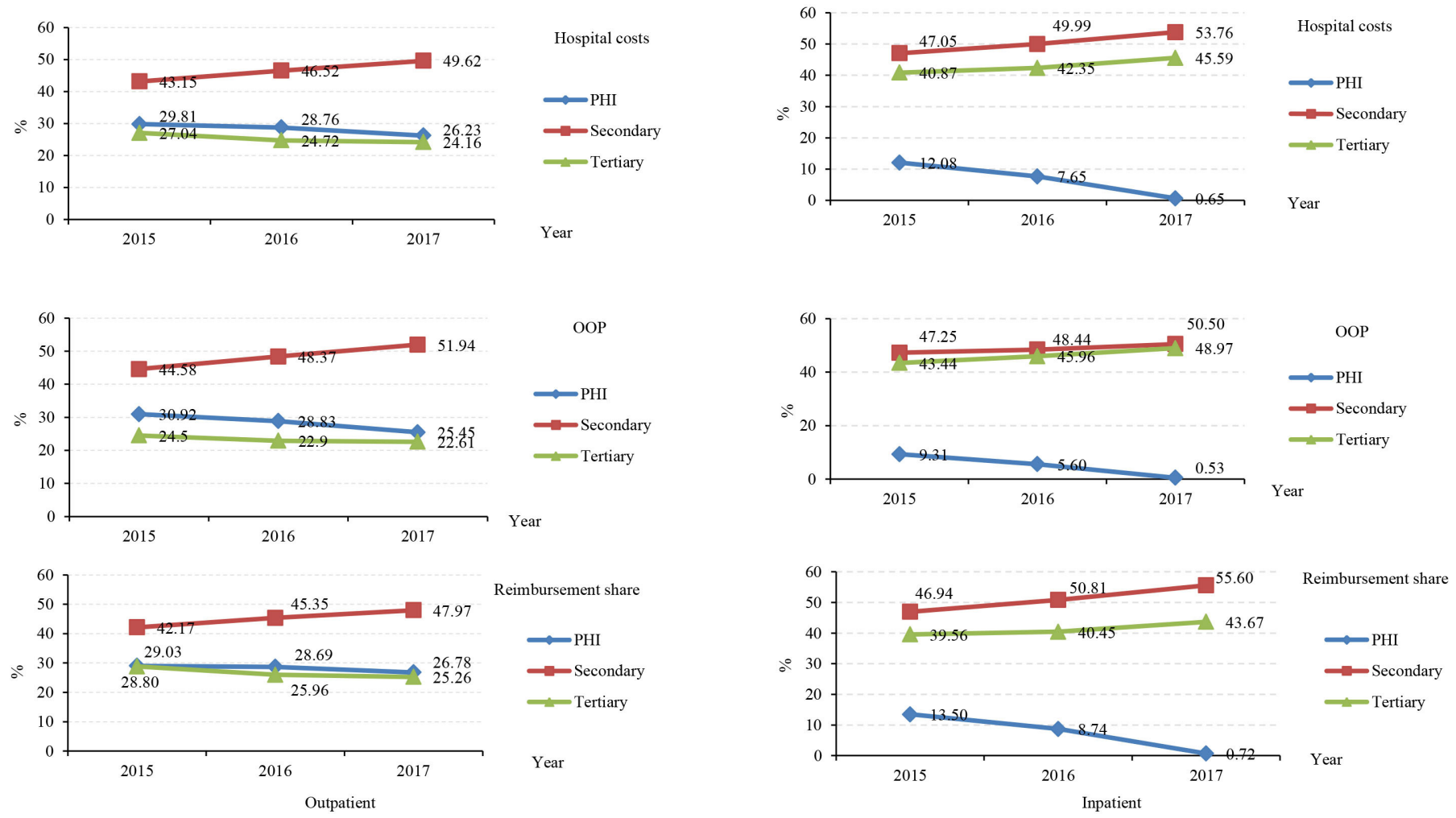

Figure 2 Share of total hospital costs, OOP expenses and reimbursements by level of hospital. OOP, out-of-pocket; PHI, primary health institution. 

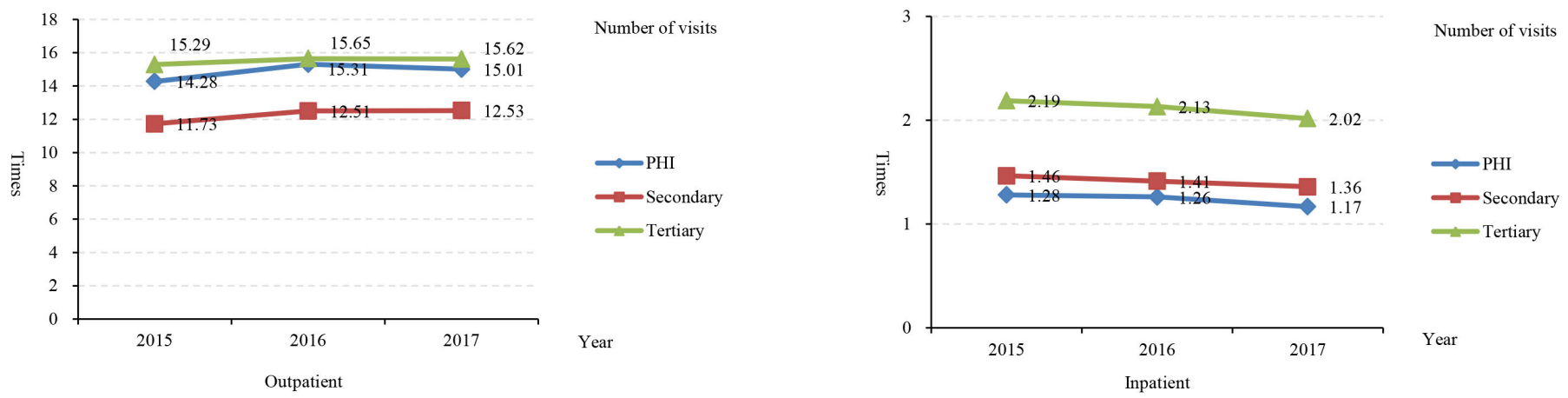

Figure 3 Average number of visits by level of hospital. PHI, primary health institution.

\section{DISCUSSION}

The hierarchical medical system has become an essential system in many countries to organise healthcare provision. ${ }^{30-32}$ As shown in figure 1, PHI outpatient and inpatient health services had lower average hospital costs and average OOP expenses than secondary-tertiary hospitals, which was consistent with previous studies. ${ }^{33}$ From the health service's perspective, the preference was for patients to use the lowest hospital cost provider, which were PHIs before and after the hierarchical system changes, and for PHIs to have the largest number and share of visits compared with secondary-tertiary hospitals. From the patients' perspective, PHIs should have been preferred since OOP expenses were lowest in PHIs, imposing a lower financial burden on patients than visits to secondary-tertiary hospitals. On average, patients also preferred more visits to hospital as a broad measure of hospital care, while the health system wanted patients to visit hospital the minimum number of times. To achieve the aim of shifting patients from high-cost secondary and tertiary hospitals to low-cost PHIs, hospital costs and OOP expenses should fall faster in PHIs, PHIs to account for a larger share of OOP expenses and hospital costs, and the number and share of PHI visits should rise faster than for secondary-tertiary hospitals. ${ }^{34}$

As show in in figure 1, OOP outpatient expenses did fall more rapidly in PHIs than in higher level hospitals, with the rate of decline of $13.7 \%$ for PHIs significantly greater than for secondary $(5.0 \%)$ and tertiary $(3.5 \%)$ hospitals. However, for inpatients, OOP expenses fell the least for
PHIs $(2.2 \%)$, followed by secondary hospitals $(5.5 \%)$ and tertiary hospitals $(7.4 \%)$, the opposite of the aim of the hierarchical system reform. Measured by hospital cost shares and visit shares, figures 2-4 show that there was no shift towards PHIs by patients with diabetes. The rate of increase in the share of total outpatient hospital costs in secondary hospitals rose $15.0 \%$, while the PHI share of total outpatient hospital costs declined $12.0 \%$, the opposite outcomes of the aim of the hierarchical system reform. For inpatients in figure 2, the increased share of hospital costs and OOP expenses reflected inpatients shifting away from PHIs towards secondary and tertiary hospitals under the hierarchical system, which indicated that the reform did not achieve the potential cost savings. ${ }^{35}$ Average outpatient visits rose roughly at the same rate in PHIs $(5.1 \%)$ as secondary hospitals $(6.8 \%)$, offering little support for the hierarchical system's aim to shift patients between hospital levels. The same conclusion holds for the fall in average inpatient visits that declined at roughly the same rate for PHIs $(9.4 \%)$ and secondary $(7.5 \%)$ and tertiary $(7.8 \%)$ hospitals. Further, the yearly regression coefficients in table 2 show that outpatients and inpatients with diabetes increasingly used higher level hospitals rather than PHIs.

Based on our outcome data, the hierarchical health reform did not shift patient utilisation towards PHIs and away from high-cost secondary-tertiary hospitals. However, in a high-income country (eg, Estonia), hierarchical reform has shifted some ambulatory care-sensitive conditions from secondary to primary care, especially patients

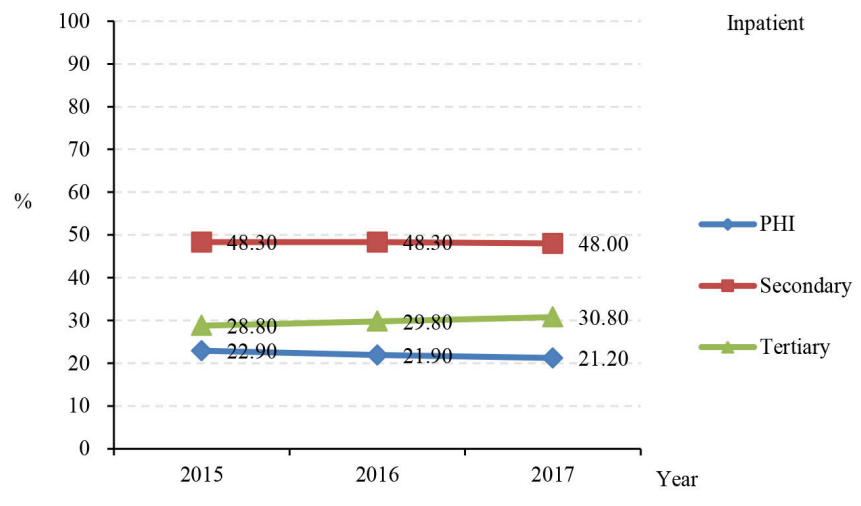

Figure 4 Share of diabetes visits by level of hospital. PHI, primary health institution. 


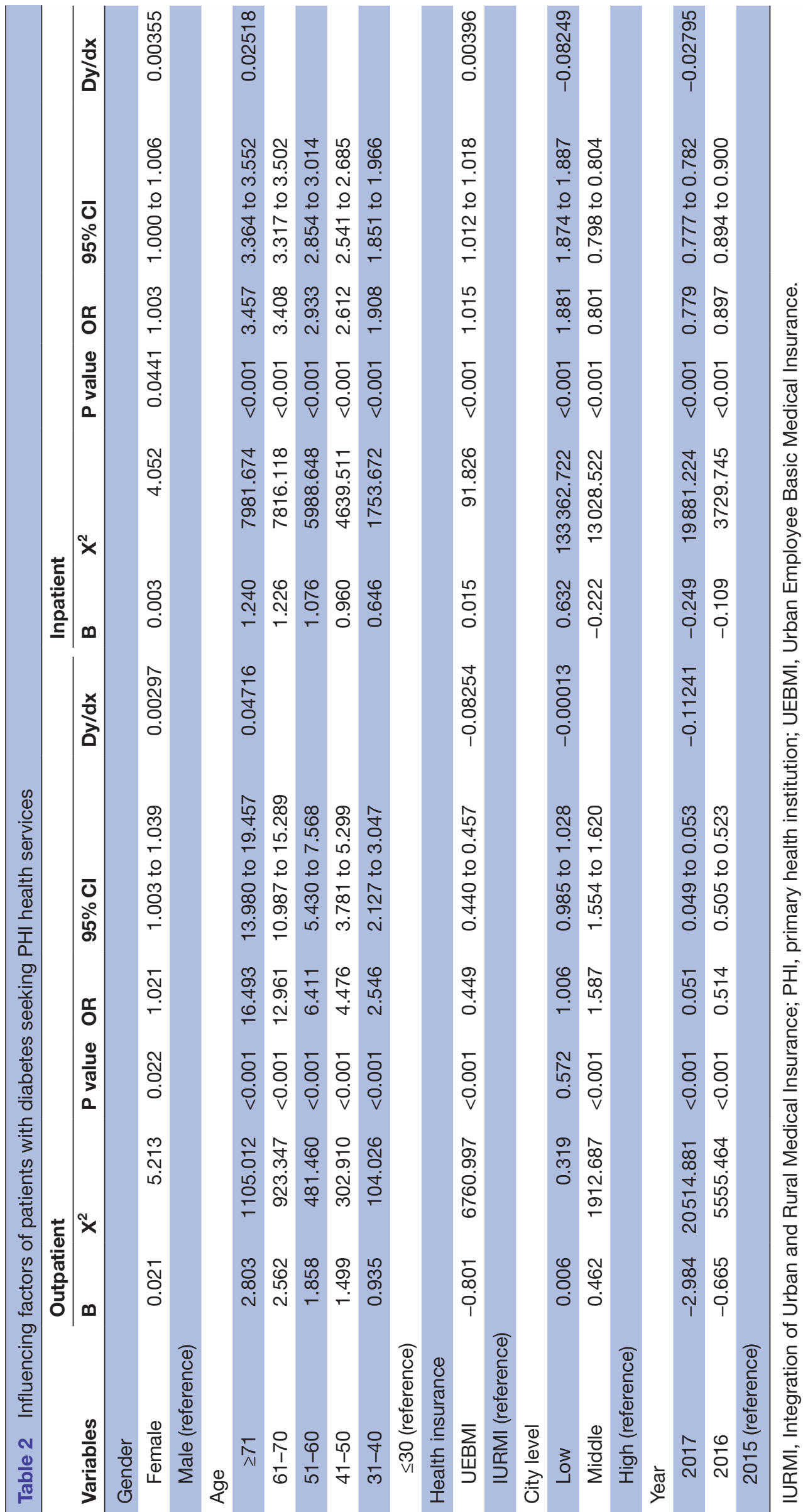


with diabetes, where healthcare utilisation increased in PHIs and fell in inpatient settings. ${ }^{29}$ This shift was mainly mediated through supply-side changes, including the introduction of family medicine and nurses working in PHIs, alongside financial changes. More generally, the high level of hospitalisation admissions in some highincome countries indicates the poor quality of PHIs, especially for the continuity of care, ${ }^{36-38}$ which should inform policy makers seeking to improve hierarchical health reform in China. In addition, research suggests that better quality of PHI care together with financial reimbursement can lead to reductions in hospitalisations for certain chronic diseases including diabetes. ${ }^{39-41}$

While diabetes can be controlled by standardised drugs and basic level management, with PHIs well suited to most diabetes treatments, our data show that patients with diabetes continued to use health services in secondary and tertiary hospitals rather than PHIs. In part, this might be a failure in the downward referral from tertiary and secondary hospitals to PHIs, which suggests that a more rigorous referral process should be instituted to relieve the pressure on higher level hospitals and encourage more diabetes medical treatments in lower cost PHIs. ${ }^{42-44}$ Perhaps patients found the price signals too weak. The hospital system might consider stronger price signals, such as much higher consultation fees and treatment charges, with lower reimbursement rates at secondarytertiary hospitals. More importantly, patients might not react only to price signals. Outpatients and inpatients need to be educated to use PHIs both for treatment and as the first stop for primary health assessment before potential referral to higher level health services rather than presenting initially at higher level hospitals. Encouraging PHI use may also require the continued investment in PHIs to improve their standard of care and to give diabetes sufferers confidence in using PHIs.

The results of binary logistic regression showed that female, older patients and those with UEBMI for outpatient services were more likely to seek health services in PHIs. Previous studies also showed that compared with male and young patients, female and older patients had a higher probability of using primary healthcare in PHIs. ${ }^{29}$ Further reforms to the hierarchical system should further encourage patients with stable diabetes, currently being treated in secondary and tertiary hospitals, to shift to PHIs. Studies also found that in spite of significant shifts between higher level hospitals and PHIs, the challenges of high levels of specialist care and long hospital stays remained, which is consistent with our results. ${ }^{45}$ Moreover, public education regarding use of PHIs as the first stop for primary health assessment should focus on young people and men in low-income cities.

\section{Implications for health policy}

Based on the findings of this study, the implications for policy makers and researchers are as follows. Considering the slight differences in reimbursement rates in figure 1 for patients with diabetes in different levels of hospitals, larger differences in reimbursement rates at different levels of hospitals are recommended. Additionally, the reimbursement difference should be regulated on the basis of health services provided rather than only on the level of hospital. For example, the reimbursement rate for the diagnosis and treatment for common chronic diseases with stable illness in PHIs should be significantly higher than that of severe acute diseases, which need to be treated in higher level hospitals. Second, because of the low healthcare utilisation of PHIs under hierarchical hospital reform, there is an urgent need to improve the capabilities of primary care delivery in PHIs, especially case management and rehabilitation care, to attract patients, especially those with chronic diabetes in a stable condition. This should encourage a higher take-up of PHI services. Third, it is necessary to strengthen collaboration between hospitals at different levels. Two-way referral channels between hospitals would promote a shift of patients towards hospitals at lower levels. Moreover, evidence showed that diabetes care collaborative teams, composed of physicians, nurses, diabetes educators, dietitians, case and care managers, discharge planners, and pharmacists, are effective in helping patients navigate safely and effectively through the hierarchical healthcare system. ${ }^{46}{ }^{47}$ Fourth, people-centred care in PHI would be helpful in building patients' trusts in PHIs, which would encourage patients to seek care in PHI as their first choice. Fifth, there is scope for further health insurance reform, to differentiate reimbursement benefits between schemes to encourage a greater take-up of PHI services. Lastly, considering the significant number of young patients with diabetes lower than 60 years, it is urgent to develop strategies to prevent or delay the occurrence of diabetes.

Turning to limitations of our study for researchers, we collected data from 11 cities in one province, and even though there were a large number of patients with diabetes included in the study, future studies should further explore the effect of hierarchical diagnosis and treatment in a much wider range of provinces. Second, the behaviour of patients with diabetes may diverge from the choices of patients with other illnesses, which might limit the generalisability of diabetes patient behaviour across the Chinese health system. Third, due to the limited variables in the health insurance bureau, some information was not available for analyses, such as the severity of diabetes and comorbidities, which may influence the choice of hospital. Future research should further discuss the behaviours of patients with diabetes with comorbidities within the hierarchical diagnosis and treatment in China. Moreover, our results were based on the data between 2015 and 2017, and a longer period of time might be required to examine the effects of hierarchical medical reform.

\section{CONCLUSION}

For patients with diabetes, hospital costs and OOP expenses fell after the introduction of the hierarchical 
medical treatment system in 2015, but this occurred across all hospital levels. While outpatient OOP expenses fell more rapidly in PHIs than in higher level hospitals (but not for inpatients), this did not lead to a shift in hospital utilisation towards PHIs. Whether measured by changes in the share of total costs, OOP expenses and reimbursements or the reimbursement rate, the hierarchical medical treatment system did not shift diabetes patient care choice towards PHIs. The rising share of total hospital costs in secondary hospitals for outpatients and in secondary and tertiary hospitals for inpatients reflected a decreased utilisation of PHIs, the opposite of the goal of the hierarchical medical system. Enhancing the utilisation of PHIs for patients with diabetes requires further hierarchical system and healthcare reform, including educating patients on PHI use, further reforming the health insurance schemes, improving PHI facilities and increasing referrals to PHIs from higher level hospitals.

\section{Author affiliations}

${ }^{1}$ Center for Health Policy Studies, School of Public Health, Zhejiang University School of Medicine, Hangzhou, China

${ }^{2}$ School of Public Health, Health Development Research Center, Sun Yat-Sen University, Guangzhou, China

${ }^{3}$ Center for Health Economic Experiments and Public Policy, Department of Social Medicine and Administration, School of Public Health, Shandong University, Jinan, China

${ }^{4}$ Dong Furen Institute of Economic and Social Development, Wuhan University,

Wuhan, China

${ }^{5}$ Center for Health Economics and Management at School of Economics and Management, Wuhan University, Wuhan, China

${ }^{6}$ Australian National Institute of Management and Commerce, Sydney, New South Wales, Australia

${ }^{7}$ Research Institute for International Strategies, Guangdong University of Foreign Studies, Guangzhou, China

${ }^{8}$ School of Economics and School of Management, Tianjin Normal University, Tianjin, China

${ }^{9}$ Newcastle Business School, University of Newcastle, University Drive, Newcastle, New South Wales, Australia

Acknowledgements The authors would like to thank the health insurance bureau for their support of the data.

Contributors All authors were responsible for the structure of this paper. JX conducted literature search and drafted the paper. SN, XW, JX, HH and JW contributed to the conception and design, interpretation of the data, and critical revisions of the paper, and approved the final version for submission.

Funding The study was partly supported by the Zhejiang Health Science and Technology Special Fund.

\section{Competing interests None declared.}

Patient and public involvement Patients and/or the public were not involved in the design, or conduct, or reporting, or dissemination plans of this research.

Patient consent for publication Not required.

Ethics approval Since the data provided by the health insurance bureaus did not identify individual patients, Dong Furen Institute of Economic and Social Development of Wuhan University ethical approval and consent were not required.

Provenance and peer review Not commissioned; externally peer reviewed.

Data availability statement All data relevant to the study are included in the article or uploaded as supplementary information. All of the main data have been included in the results. Additional materials with details may be obtained from the corresponding author.

Open access This is an open access article distributed in accordance with the Creative Commons Attribution Non Commercial (CC BY-NC 4.0) license, which permits others to distribute, remix, adapt, build upon this work non-commercially, and license their derivative works on different terms, provided the original work is properly cited, appropriate credit is given, any changes made indicated, and the use is non-commercial. See: http://creativecommons.org/licenses/by-nc/4.0/.

\section{ORCID iDs}

Xin Wang http://orcid.org/0000-0001-6588-276X

Jian Wang http://orcid.org/0000-0001-6596-0684

\section{REFERENCES}

1 International Diabetes Federation. IDF diabetes atlas. 9th edn, 2019. https://diabetesatlas.org/en/sections/demographic-and-geographicoutline.html

2 Institute for Health Metrics and Evaluation. GBD 2016. University of Washington. Available: https://vizhub.healthdata.org/gbd-compare/

3 Chen D, Wang M-W. Development and application of rodent models for type 2 diabetes. Diabetes Obes Metab 2005;7:307-17.

4 Yang W, Lu J, Weng J, et al. Prevalence of diabetes among men and women in China. N Engl J Med 2010;362:1090-101.

$5 \mathrm{Hu} \mathrm{H}$, Sawhney M, Shi L, et al. A systematic review of the direct economic burden of type 2 diabetes in China. Diabetes Ther 2015;6:7-16.

6 Yao Q, Han X, Ma X-K, et al. Cloud-based hospital information system as a service for grassroots healthcare institutions. J Med Syst 2014;38:104.

7 Wu T-Y, Majeed A, Kuo KN. An overview of the healthcare system in Taiwan. London J Prim Care 2010;3:115-9.

8 Zhang Y, Feng $\mathrm{X}$. The relationship between job satisfaction, burnout, and turnover intention among physicians from urban state-owned medical institutions in Hubei, China: a cross-sectional study. BMC Health Serv Res 2011;11:235.

9 Liu Y, Zhang L, Yang HQ. Does the increase of the numbers of medical institutions can solve the problem of "difficult and expensive to see a doctor"? Medicine \& Philosophy 2016.

10 Pan J, Liu D, Ali S. Patient dissatisfaction in China: what matters. Soc Sci Med 2015;143:145-53.

11 Liu Y, Yuan Z, Liu Y, et al. Changing community health service delivery in economically less-developed rural areas in China: impact on service use and satisfaction. BMJ Open 2014;4:e004148.

12 Yan Z, Wan D, Li L. Patient satisfaction in two Chinese provinces: rural and urban differences. Int J Qual Health Care 2011;23:384-9.

13 The Lancet. Health-Care system transition in China. Lancet 2018;391:1332.

14 Meng Q, Yuan J, Hou ZY. Service and function analysis of grassroots health institutions in China. Health Policy Anal Chin 2009;2:1-6.

15 Wang Y, Sun L, Hou J. Hierarchical medical system based on big data and mobile Internet: a new strategic choice in health care. JMIR Med Inform 2017;5:e22.

16 Report on the distribution and use of financial medical and health funds. Available: vhttp://www.gov.cn/xinwen/2018-12/27/content_ 5352845.htm

17 Liu L, Johnson HL, Cousens S, et al. Child health epidemiology reference group of who and UNICEF. global, regional, and national causes of child mortality: an updated systematic analysis for 2010 with time trends since 2000. Lancet 2012;379:2151-61.

18 Pan J, Hu J, Cui H. Capacity of primary hospitals for diabetes treatment in six Chinese provinces: a cross-sectional survey. Lancet Diabet Endocrinol 2016;4:S10.

19 National Health Commission of the People's Republic of China. China health statistics Yearbook, 2019. Beijing: China Union Medical University Press.

20 Porter ME, Lee TH. The strategy that will fix healthcare. Harv Bus Rev 2013:91:50-70

21 van Ginneken E. Perennial health care reform - the long Dutch quest for cost control and quality improvement. $N$ Engl J Med 2015;373:885-9.

22 Schäfer W, Kroneman M, Boerma W, et al. The Netherlands: health system review. Health Syst Transit 2010;12:1-229.

23 Rijksoverheid. Naar beter betaalbare zorg. Rapport Taskforce Beheersing Zorguitgaven . To more affordable care. Report Taskforce Control Healthcare Expenditures.] Ministerie van Volksgezondheid, Welzijn en Sport; Den Haag: Rijksoverheid 2012.

24 Vlek JF, Vierhout WP, Knottnerus JA. A randomised controlled trial of joint consultations with general practitioners and cardiologists in primary care. Br J Gen Pract 2003;53:108-12.

25 Black M, Leese B, Gosden T, et al. Specialist outreach clinics in general practice: what do they offer? Br J Gen Pract 1997;47:558-61. 
26 Bowling A, Bond M. A national evaluation of specialists' clinics in primary care settings. Br J Gen Pract 2001;51:264-9.

27 Pan J, Tian S, Zhou Q, et al. Benefit distribution of social health insurance: evidence from china's urban resident basic medical insurance. Health Policy Plan 2016;31:czv141

$28 \mathrm{Xu} \mathrm{J}$, Wang J, King M, et al. Rural-urban disparities in the utilization of mental health inpatient services in China: the role of health insurance. Int J Health Econ Manag 2018;18:377-93.

29 Atun R, Gurol-Urganci I, Hone T, et al. Shifting chronic disease management from hospitals to primary care in Estonian health system: analysis of national panel data. $J$ Glob Health 2016;6:020701.

30 Goldfield N, Gnani S, Majeed A. Primary care in the United States: profiling performance in primary care in the United States. BMJ 2003:326:744-7.

31 Palmer G, Reid B. Evaluation of the performance of diagnosis-related groups and similar casemix systems: methodological issues. Health Serv Manage Res 2001;14:71-81.

32 Pettoello-Mantovani M, Namazova-Baranova L, Ehrich J. Integrating and rationalizing public healthcare services as a source of cost containment in times of economic crises. Ital J Pediatr 2016;42:18.

33 Winpenny E, Miani C, Pitchforth E, et al. Outpatient services and primary care: scoping review, substudies and international comparisons. Health Services and Delivery Research 2016;4:1-290.

34 Quanjel TCC, Struijs JN, Spreeuwenberg MD, et al. Shifting hospital care to primary care: an evaluation of cardiology care in a primary care setting in the Netherlands. BMC Fam Pract 2018;19:55.

35 Dusheiko M, Gravelle H, Martin S, et al. Does better disease management in primary care reduce hospital costs? Evidence from English primary care. $J$ Health Econ 2011;30:919-32.
36 Tian Y, Dixon A, Gao H. Emergency hospital admissions for ambulatory care-sensitive conditions: identifying the potential for reductions. London: King's Fund, 2012.

37 Purdy S. Avoiding hospital admissions: what does the research evidence say? London: King's Fund, 2010.

38 Huntley A, Lasserson D, Wye $L$, et al. Which features of primary care affect unscheduled secondary care use? A systematic review. BMJ Open 2014;4:e004746

39 Bottle A, Millett C, Xie Y, et al. Quality of primary care and hospital admissions for diabetes mellitus in England. J Ambul Care Manage 2008;31:226-38.

40 Lippi Bruni M, Nobilio L, Ugolini C. Economic incentives in general practice: the impact of pay-for-participation and pay-for-compliance programs on diabetes care. Health Policy 2009;90:140-8

41 Dusheiko M, Doran T, Gravelle H, et al. Does higher quality of diabetes management in family practice reduce unplanned hospital admissions? Health Serv Res 2011;46:27-46.

42 Xiang L, Li N, Liu C. Urban-rural disparity in utilization of preventive care services in China. Medicine 2016;95:e4783.

43 Wu D, Lam TP, Lam KF. Health reforms in China: the public's choices for first-contact care in urban areas. Fam Pract 2017:34:194-200.

44 Chen Z. Launch of the health-care reform plan in China. Lancet 2009;373:1322-4.

45 World Bank Group. The state of health care integration in Estonia: summary report Tallinn, Estonia: Estonian health insurance fund (EHIF), 2015. Available: http://www.haigekassa.ee/sites/default/files/ Maailmapangauuring/summary_report_hk_2015.pdf

46 Sibbald B, McDonald R, Roland M. Shifting care from hospitals to the community: a review of the evidence on quality and efficiency. $J$ Health Serv Res Policy 2007;12:110-7.

47 Rogers S. Inpatient care coordination for patients with diabetes. Diabetes Spectrum 2008;21:272-5. 